\title{
Effect of insecticide on the population dynamics of brown planthoppers and Cyrtorhinus lividipennis: a modeling approach
}

Inthira Chaiya ${ }^{1}$ and Chontita Rattanaku1 ${ }^{2,3^{*}}$ (D)

"Correspondence:

chontita.rat@mahidol.ac.th

${ }^{2}$ Center of Excellence in

Mathematics, Commission on

Higher Education, Bangkok,

Thailand

${ }^{3}$ Department of Mathematics,

Faculty of Science, Mahidol

University, Bangkok, Thailand

Full list of author information is

available at the end of the article

\section{Springer}

\begin{abstract}
In this paper, we investigate the effect of insecticide on the population dynamics of brown planthoppers (a major insect pest of rice) and Cyrtorhinus lividipennis (one of the natural enemies of brown planthoppers) by developing an impulsive mathematical model, and we analyze the model theoretically and numerically. The conditions on the parameters of the model for which the stability and permanence of the system can be ensured are derived. Computer simulations are also presented to confirm our theoretical results. The results show that with an appropriate amount and period between two consecutive applications of insecticide, the population of brown planthoppers could be maintained below a certain level, while Cyrtorhinus lividipennis could also survive.
\end{abstract}

Keywords: Mathematical model; Brown planthopper; Cyrtorhinus lividipennis; Insecticide

\section{Introduction}

The brown planthopper (BPH) is recognized as one of the major insect pests of rice. The well-known damage caused by the infestation of brown planthoppers is hopperburn in which the rice crop are wilting and drying completely [1]. The outbreak of BPH in Thailand during the dry season of the year 2010 caused approximately $\$ 52$ million losses as reported by the Office of Agricultural Economics, the Ministry of Agriculture and Cooperatives of Thailand [2].

Biological control and insecticide have been used to control the outbreak of brown planthoppers in a paddy field. However, when insecticide is utilized not only brown planthoppers are eliminated but also its natural enemies in the paddy field such as Cyrtorhinus lividipennis [3, 4]. Even though insecticides have been widely used for controlling the pest, BPH has developed resistance to some major insecticides such as carbamates, organophosphates, neonicotinoids, phenylpyrazoles and pyrethroids [5, 6]. As it is quick and cost-effective against insects, chemical control is a popular choice in pest management. However, excessive and irrational use of chemical pesticides could lead to negative effects on the environment such as biodiversity's reduction and the decrease in population of natural enemies. Alternatively, biological control is a safe and an effective method. Cyrtorhinus Lividipennis is a major natural enemy of $\mathrm{BPH}$. It preys mainly on eggs and nymphs

(c) The Author(s) 2019. This article is distributed under the terms of the Creative Commons Attribution 4.0 International License (http://creativecommons.org/licenses/by/4.0/), which permits unrestricted use, distribution, and reproduction in any medium, provided you give appropriate credit to the original author(s) and the source, provide a link to the Creative Commons license, and indicate if changes were made. 
of BPH [7, 8]. The predatory activity of Cyrtorhinus lividipennis against BPH has been investigated by many researchers and the study indicated that the Cyrtorhinus lividipennis's preying on BPH's eggs was an important cause of the decrease in the BPH population [7, 9]. However, when the outbreak of BPH is severe, the use of Cyrtorhinus lividipennis alone might not be the most effective choice because the reproduction of Cyrtorhinus lividipennis is not rapid enough to control the outbreak.

In this paper, we investigate the effects of impulsive applications of insecticide on the population dynamics of brown planthoppers and Cyrtorhinus lividipennis.

\section{An impulsive system}

Let $B(t)$ represent the population density of brown planthoppers at time $t$ and $C(t)$ represent the population density of Cyrtorhinus lividipennis at time $t$. The following impulsive system is proposed to investigate the population dynamics of brown planthoppers and Cyrtorhinus lividipennis when insecticide is utilized:

$$
\left.\begin{array}{l}
\frac{d B}{d t}=a_{1} B\left(1-\frac{B}{h_{1}}\right)-\frac{b_{1} B C}{1+h_{2} B}-d_{1} B \\
\frac{d C}{d t}=a_{2} C\left(1-\frac{C}{h_{3}}\right)+\frac{r_{1} b_{1} B C}{1+h_{2} B}-d_{2} C
\end{array}\right\} t \neq k T,
$$

with

$$
\left.\begin{array}{l}
B\left(t^{+}\right)=(1-\alpha) B(t) \\
C\left(t^{+}\right)=(1-\beta) C(t)
\end{array}\right\} \quad t=k T .
$$

Here, $a_{1}, a_{2}, b_{1}, r_{1}, h_{1}, h_{2}, h_{3}, d_{1}$ and $d_{2}$ are assumed to be positive, $T$ accounts for the period between two consecutive applications of insecticide, $k \in Z_{+}, Z_{+}=\{1,2,3, \ldots\}, \alpha$ accounts for the negative effect of the insecticide on the population of brown planthoppers, $0<\alpha<1$, and $\beta$ accounts for the negative effect of insecticide on the population of Cyrtorhinus lividipennis, $0<\beta<1$.

Equation (1a) describes the rate of change of the population of brown planthoppers. On the right-hand side, the first term represents the reproduction of brown planthoppers which is assumed to follow the logistic growth function, the second term represents the decrease in the population of brown planthoppers due to the predation by Cyrtorhinus lividipennis when the functional response is assumed to follow the Holling type II functional response and the last term represents the natural death rate of brown planthoppers.

Equation (1b) describes the rate of change of the population of Cyrtorhinus lividipennis. On the right-hand side, the first term represents the reproduction of Cyrtorhinus lividipennis which is assumed to follow the logistic growth function. Here, we assume that Cyrtorhinus lividipennis could feed on other insect pest in the paddy field apart from brown planthoppers and the population of Cyrtorhinus lividipennis could be high even as brown planthoppers are absent as reported in [10]. The second term represents the increase in the population of Cyrtorhinus lividipennis due to the predation on brown planthoppers and the last term represents the natural death rate of Cyrtorhinus lividipennis. 


\section{Theoretical results}

Definition 1 Let the map defined by the right hand side of (1a)-(1d) be denoted by $f=$ $\left(f_{1}, f_{2}\right)$ and let $G: R_{+} \times R_{+}^{2} \rightarrow R_{+}$, where $R_{+}=[0, \infty), R_{+}^{2}=\left\{X \in R^{2}: X=(B, C), B \geq 0, C \geq 0\right\}$.

(a) $G$ is said to belong to class $G_{0}$ if $G$ is continuous in $(k T,(k+1) T] \times R_{+}^{2} \rightarrow R_{+}$and for each $X \in R_{+}^{2}, k \in Z_{+}$,

$$
\lim _{(t, Y) \rightarrow\left(k T^{+}, X\right)} G(t, Y)=G\left(k T^{+}, X\right)
$$

exists and $G$ is locally Lipschitzian in $X$.

(b) Suppose $G \in G_{0}$. For $(t, X) \in(k T,(k+1) T] \times R_{+}^{2}$, the upper right derivative of $G(t, X)$ with respect to (1a)-(1d) is defined by

$$
D^{+} G(t, X)=\limsup _{h \rightarrow 0^{+}} \frac{1}{h}[G(t+h, X+h f(t, X))-G(t, X)] .
$$

The solution of (1a)-(1d), $X(t)=(B(t), C(t))$, is assumed to be a piecewise continuous function in what follows. This implies that $X(t): R_{+} \rightarrow R_{+}^{2}, X(t)$ is continuous on $(k T,(k+$ 1) $T$ ], $k \in Z_{+}$and $\lim _{t \rightarrow k T^{+}} X(t)=X\left(k T^{+}\right)$exists. Therefore, the smoothness properties of $f$ ensure the existence and uniqueness of solution to (1a)-(1d).

Consider (1a) and (1b) when $t \neq k T$. We can see that if $B(t)=0, \frac{d B}{d t}=0$ and if $C(t)=0$, $\frac{d C}{d t}=0$. In addition, $B\left(k T^{+}\right)=(1-\alpha) B(k T), 0<\alpha<1, C\left(k T^{+}\right)=(1-\beta) C(k T), 0<\beta<1$. Hence, the following lemma is obtained.

Lemma 1 Let $X(t)=(B(t), C(t))$ be a solution of (1a)-(1d). Then $X(t) \geq 0$ for all $t \geq 0$ provided that $X\left(0^{+}\right) \geq 0$.

Lemma 2 Let $X(t)=(B(t), C(t))$ be a solution of $(1 \mathrm{a})-(1 \mathrm{~d})$. Then $B(t) \leq M^{*}$ and $C(t) \leq M^{*}$ for some constant $M^{*}>0$ provided that

$$
d_{2}>\frac{r_{1} b_{1}}{h_{2}}
$$

when $t$ is sufficiently large.

Proof

Let $g(t)=B(t)+C(t), M_{1}=\frac{a_{1} h_{1}}{4}, M_{2}=\frac{a_{2} h_{3}}{4}, M_{3}=\frac{r_{1} b_{1}}{h_{2}}$ and $c^{*}=\min \left\{d_{1}, d_{2}-M_{3}\right\}$.

For $t \neq k T$, we can see that

$$
\begin{aligned}
D^{+} g & +c^{*} g \\
& =\frac{d B}{d t}+\frac{d C}{d t}+c^{*} B+c^{*} C \\
& =a_{1} B\left(1-\frac{B}{h_{1}}\right)-\frac{b_{1} B C}{1+h_{2} B}-d_{1} B+a_{2} C\left(1-\frac{C}{h_{3}}\right)+\frac{r_{1} b_{1} B C}{1+h_{2} B}-d_{2} C+c^{*} B+c^{*} C \\
& \leq\left(c^{*}-d_{1}\right) B+\left(c^{*}-d_{2}+M_{3}\right) C+M_{1}+M_{2} \\
& \leq M_{1}+M_{2} \equiv M_{0} .
\end{aligned}
$$

Hence, $D^{+} g \leq-c^{*} g+M_{0}$. 
For $t=k T$,

$$
\begin{aligned}
g\left(k T^{+}\right) & =B\left(k T^{+}\right)+C\left(k T^{+}\right)=(1-\alpha) B(k T)+(1-\beta) C(k T) \\
& =B(k T)+C(k T)-\alpha B(k T)-\beta C(k T) \\
& \leq g(k T) .
\end{aligned}
$$

For $t \in(k T,(k+1) T]$, Lemma 2.2 of [11] implies that

$$
\begin{aligned}
g(t) & \leq g(0) e^{-c^{*} t}+\int_{0}^{t} M_{0} e^{-c^{*}(t-x)} d x \\
& \leq g(0) e^{-c^{*} t}+M_{0}\left(\frac{1}{c^{*}}-\frac{e^{-c^{*} t}}{c^{*}}\right) \\
& <\frac{M_{0}}{c^{*}} \equiv M^{*} \quad \text { as } t \rightarrow \infty .
\end{aligned}
$$

Since $g(t)=B(t)+C(t)$ and $g(t)<M^{*}$, this means that $B(t) \leq M^{*}$ and $C(t) \leq M^{*}$ when $t$ is large enough and $M^{*}>0$.

Next, let us investigate the reduced system of (1a)-(1d) when the brown planthopper is absent $(B=0)$ :

$$
\begin{aligned}
& \frac{d C}{d t}=s C-r C^{2}, \quad t \neq k T, \\
& C\left(k T^{+}\right)=(1-\beta) C(k T), \quad t=k T, \\
& C\left(0^{+}\right)=C_{0},
\end{aligned}
$$

where $r \equiv \frac{a_{2}}{h_{3}}>0$ and $s \equiv a_{2}-d_{2}$. Suppose that $s>0$ that is

$$
a_{2}>d_{2} \text {. }
$$

We can see that the solution of (3) is

$$
C(t)=\frac{s}{r+c_{1} s e^{-s t}},
$$

where $c_{1}$ is an arbitrary constant.

Since $C(t)$ is an increasing function for $s>0$ and (4), the system (3)-(5) has a periodic solution

$$
\left.\tilde{C}(t)=\frac{s\left(1-\beta-e^{-s T}\right)}{\beta r e^{-s(t-k T)}+r\left(1-\beta-e^{-s T}\right)}\right), \quad t \in(k T,(k+1) T],
$$

with $\tilde{C}\left(0^{+}\right)=\frac{s\left(1-\beta-e^{-s T}\right)}{r\left(1-e^{-s T}\right)}>0$ and (6) holding.

Therefore,

$$
C(t)=\frac{C_{0} s\left(1-\beta-e^{-s T}\right) \tilde{C}(t) e^{s t}}{\left[s\left(1-\beta-e^{-s T}\right)-C_{0} r\left(1-e^{-s T}\right)\right] \tilde{C}(t)+C_{0} s\left(1-\beta-e^{-s T}\right) e^{s t}}, \quad t \in(k T,(k+1) T],
$$

is the positive solution of (3)-(5). 
Lemma 3 The system (3)-(5) has a positive periodic solution $\tilde{C}(t)$, and $C(t) \rightarrow \tilde{C}(t)$ as $t \rightarrow \infty$ for every solution $C(t)$ of (3)-(5).

Therefore,

$$
(0, \tilde{C}(t))=\left(0, \frac{s\left(1-\beta-e^{-s T}\right)}{\beta r e^{-s(t-k T)}+r\left(1-\beta-e^{-s T}\right)}\right)
$$

is a periodic solution of the system (1a)-(1d) at the absence of brown planthoppers for $t \in(k T,(k+1) T]$ and $\tilde{C}\left(k T^{+}\right)=\tilde{C}\left(0^{+}\right)=\frac{s\left(1-\beta-e^{-s T}\right)}{r\left(1-e^{-S T}\right)}, k \in Z_{+}$.

\section{Theorem 1 Suppose that}

$$
\begin{aligned}
& T_{2}<T<T_{1}, \\
& a_{1}>d_{1}+\frac{b_{1} s}{r},
\end{aligned}
$$

and

$$
\ln \left(\frac{1}{1-\alpha}\right)>\frac{b_{1}}{r} \ln \left(\frac{1}{1-\beta}\right)
$$

Then the solution $(0, \tilde{C}(t))$ of $(1 \mathrm{a})-(1 \mathrm{~d})$ is locally asymptotically stable where $T_{1}=$ $\frac{1}{\left(a_{1}-d_{1}-\frac{b_{1} s}{r}\right)}\left[\ln \left(\frac{1}{1-\alpha}\right)-\frac{b_{1}}{r} \ln \left(\frac{1}{1-\beta}\right)\right]$ and $T_{2}=\frac{1}{s} \ln \left(\frac{1}{1-\beta}\right)$.

Proof Let us consider a small perturbation

$$
\begin{aligned}
& B(t)=v_{1}(t), \\
& C(t)=\tilde{C}(t)+v_{2}(t),
\end{aligned}
$$

from the point $(0, \tilde{C}(t))$. Then

$$
\left(\begin{array}{l}
v_{1}(t) \\
v_{2}(t)
\end{array}\right)=\Phi(t)\left(\begin{array}{l}
v_{1}(0) \\
v_{2}(0)
\end{array}\right), \quad 0<t<T
$$

where $\Phi(t)$ satisfies

$$
\frac{d \Phi(t)}{d t}=\left(\begin{array}{cc}
a_{1}-d_{1}-b_{1} \tilde{C}(t) & 0 \\
* & s-2 r \tilde{C}(t)
\end{array}\right) \Phi(t)
$$

and $\Phi(0)=I$, the identity matrix. Hence, the fundamental solution matrix is

$$
\Phi(t)=\left(\begin{array}{cc}
\exp \int_{0}^{t}\left(a_{1}-d_{1}-b_{1} \tilde{C}(x)\right) d x & 0 \\
* * & \exp \int_{0}^{t}(s-2 r \tilde{C}(x)) d x
\end{array}\right) .
$$

Note that the terms $(*)$ and $(* *)$ are not involved in the further calculations and hence it is not necessary to find $(*)$ and $(* *)$. 
Linearization of (1c)-(1d) yields

$$
\left(\begin{array}{l}
v_{1}\left(k T^{+}\right) \\
v_{2}\left(k T^{+}\right)
\end{array}\right)=\left(\begin{array}{cc}
1-\alpha & 0 \\
0 & 1-\beta
\end{array}\right)\left(\begin{array}{l}
v_{1}(k T) \\
v_{2}(k T)
\end{array}\right)
$$

Consider

$$
A=\left(\begin{array}{cc}
1-\alpha & 0 \\
0 & 1-\beta
\end{array}\right) \Phi(T) .
$$

The eigenvalues of $A$ are

$$
\begin{aligned}
\lambda_{1} & =(1-\alpha) \exp \int_{0}^{T}\left(a_{1}-d_{1}-b_{1} \tilde{C}(x)\right) d x \\
& =(1-\alpha) \exp \left(\left(a_{1}-d_{1}\right) T-\frac{b_{1}}{r}(\ln (1-\beta)+s T)\right), \\
\lambda_{2} & =(1-\beta) \exp \int_{0}^{T}(s-2 r \tilde{C}(x)) d x=(1-\beta) \exp (-s T-2 \ln (1-\beta)) .
\end{aligned}
$$

Since $0<\alpha<1,0<\beta<1$, (8)-(10) hold, and then

$$
\frac{1}{s} \ln \left(\frac{1}{1-\beta}\right)<T<\frac{1}{\left(a_{1}-d_{1}-\frac{b_{1} s}{r}\right)}\left[\ln \left(\frac{1}{1-\alpha}\right)-\frac{b_{1}}{r} \ln \left(\frac{1}{1-\beta}\right)\right] .
$$

Hence,

$$
\left|\lambda_{1}\right|=(1-\alpha) \exp \int_{0}^{T}\left(a_{1}-d_{1}-b_{1} \tilde{C}(x)\right) d x<1
$$

and

$$
\left|\lambda_{2}\right|=(1-\beta) \exp (-s T-2 \ln (1-\beta))<1 .
$$

All conditions of Floquet theory are now satisfied and, hence, we can conclude that the solution $(0, \tilde{C}(t))$ of (1a)-(1d) is locally stable, which completes the proof.

\section{System Permanence}

Definition 2 If there exist constants $n, m>0$ and a finite time $t_{0}$ such that, for all solution with all initial values $B\left(0^{+}\right)>0$ and $C\left(0^{+}\right)>0$,

$$
\begin{aligned}
& n \leq B(t) \leq m, \\
& n \leq C(t) \leq m,
\end{aligned}
$$

for all $t>t_{0}$, the system (1a)-(1d) is said to be permanent where we note that $t_{0}$ may depend on the initial values whereas $n, m$ are independent of the initial values. 
Theorem 2 The system (1a)-(1d) is permanent if

$$
T>T^{*}
$$

and

$$
r>s+M_{3}
$$

provided (2), (6), (9) and (10) hold where

$$
T^{*} \equiv \frac{1}{\left(a_{1}-d_{1}-\frac{b_{1}\left(s+M_{3}\right)}{r}\right)}\left[\ln \left(\frac{1}{1-\alpha}\right)-\frac{b_{1}}{r} \ln \left(\frac{1}{1-\beta}\right)\right] .
$$

Proof Let $X(t)=(B(t), C(t))$ be a solution of the system (1a)-(1d) with $B\left(0^{+}\right)>0$ and $C\left(0^{+}\right)>0$. For sufficiently large $t$, Lemma 2 implies that a constant $m>0$ exists so that $B(t) \leq m$ and $C(t) \leq m$.

Since $\frac{r_{1} b_{1} B C}{1+k_{2} B} \geq 0$, (1b) implies that

$$
\begin{aligned}
& \frac{d C}{d t} \geq s y-r y^{2}, \quad t \neq k T, \\
& C\left(k T^{+}\right)=(1-\beta) C(k T), \quad t=k T,
\end{aligned}
$$

and for sufficiently large $t$, we have

$$
C(t)>\tilde{C}(t)-\varepsilon
$$

for some $\varepsilon>0$.

Hence, for sufficiently large $t$, we obtain

$$
C(t)>\frac{s\left(1-\beta-e^{-s T}\right)}{r\left(1-e^{-s T}\right)}-\varepsilon \equiv n_{1} .
$$

Next, we have to show that there exists a constant $n_{2}>0$ such that $B(t)>n_{2}$. For some $n_{3}>0$, let

$$
\hat{M}_{1}=a_{1}\left(1-\frac{n_{3}}{h_{1}}\right)-d_{1} .
$$

Step 1. In order to prove by contradiction that there exists $t_{1}$ such that $B\left(t_{1}\right) \geq n_{3}$, we assume that $B(t)<n_{3}$ for all positive $t$.

Equations (1b) and (1d) imply that

$$
\begin{aligned}
\frac{d C}{d t} & =a_{2} C\left(1-\frac{C}{h_{3}}\right)+\frac{r_{1} b_{1} B C}{1+h_{2} B}-d_{2} C, \quad t \neq k T \\
& \leq a_{2} C\left(1-\frac{C}{h_{3}}\right)+M_{3} C-d_{2} C \\
& =\left(s+M_{3}\right) C-r C^{2},
\end{aligned}
$$




$$
C\left(t^{+}\right)=(1-\beta) C(t), \quad t=k T \text {. }
$$

Consider the comparison system

$$
\begin{aligned}
& \frac{d Z}{d t}=\left(s+M_{3}\right) Z-r Z^{2}, \quad t \neq k T, \\
& Z\left(t^{+}\right)=(1-\beta) Z(t), \quad t=k T,
\end{aligned}
$$

and

$$
Z\left(0^{+}\right)=C\left(0^{+}\right)
$$

Hence,

$$
\frac{1}{\tilde{Z}(t)}=\frac{\beta r e^{-\left(s+M_{3}\right)(t-k T)}}{\left(s+M_{3}\right)\left(1-\beta-e^{-\left(s+M_{3}\right) T}\right)}+\frac{r}{\left(s+M_{3}\right)}, \quad t \in(k T,(k+1) T],
$$

is a periodic solution of $(13)-(15)$ with $\frac{1}{Z\left(0^{+}\right)}=\frac{\beta r}{\left(s+M_{3}\right)\left(1-\beta-e^{-\left(s+M_{3}\right) T}\right)}+\frac{r}{\left(s+M_{3}\right)}>0$. The positive solution of (13)-(15) is

$$
\frac{1}{Z(t)}=\left(\frac{1}{Z\left(0^{+}\right)}-\frac{\beta r}{\left(s+M_{3}\right)\left(1-\beta-e^{-\left(s+M_{3}\right) T}\right)}-\frac{r}{\left(s+M_{3}\right)}\right) e^{-\left(s+M_{3}\right) t}+\frac{1}{\tilde{Z}(t)},
$$

$t \in(k T,(k+1) T]$ and as $t \rightarrow \infty$

$$
\frac{1}{Z(t)} \rightarrow \frac{1}{\tilde{Z}(t)}=\frac{\beta r e^{-\left(s+M_{3}\right)(t-k T)}}{\left(s+M_{3}\right)\left(1-\beta-e^{-\left(s+M_{3}\right) T}\right)}+\frac{r}{\left(s+M_{3}\right)}
$$

Hence, we can conclude that $C(t) \leq Z(t)$ by the comparison theorem [12].

Now, we consider (1a)

$$
\begin{aligned}
\frac{d B}{d t} & =a_{1} B\left(1-\frac{B}{h_{1}}\right)-\frac{b_{1} B C}{1+h_{2} B}-d_{1} B \\
& \geq\left(a_{1}\left(1-\frac{n_{3}}{h_{1}}\right)-b_{1} C-d_{1}\right) B \\
& =\left(\hat{M}_{1}-b_{1} C\right) B .
\end{aligned}
$$

Since $C(t) \leq Z(t)$, there is a $T_{1}>0$ such that

$$
C(t) \leq Z(t)<\tilde{Z}(t)+\varepsilon_{1}, \quad t \neq k T, t \geq T_{1},
$$

for a sufficiently small $\varepsilon_{1}>0$.

Therefore,

$$
\frac{d B}{d t}>\left(\hat{M}_{1}-b_{1}\left(\tilde{Z}(t)+\varepsilon_{1}\right)\right) B, \quad t \neq k T, t \geq T_{1},
$$

and

$$
B\left(t^{+}\right)=(1-\alpha) B(t), \quad t=k T, t \geq T_{1} .
$$


Letting $K \in Z_{+}$and $K T \geq T_{1}$, and integrating over $(k T,(k+1) T], k \geq K$, we get

$$
\begin{aligned}
B((k+1) T) & \geq B(k T)(1-\alpha) \exp \left(\int_{k T}^{(k+1) T}\left(\hat{M}_{1}-b_{1}\left(\tilde{Z}(t)+\varepsilon_{1}\right)\right) d t\right) \\
& =B(k T)(1-\alpha) \exp \left(\left(\hat{M}_{1}-b_{1} \varepsilon_{1}-\frac{b_{1}\left(s+M_{3}\right)}{r}\right) T+\frac{b_{1}}{r} \ln \left(\frac{1}{1-\beta}\right)\right) \\
& =B(k T) \gamma,
\end{aligned}
$$

where $\gamma \equiv(1-\alpha) \exp \left(\left(\hat{M}_{1}-b_{1} \varepsilon_{1}-\frac{b_{1}\left(s+M_{3}\right)}{r}\right) T+\frac{b_{1}}{r} \ln \left(\frac{1}{1-\beta}\right)\right)$.

Consider

$$
\ln \gamma=\ln (1-\alpha)+\left(\hat{M}_{1}-b_{1} \varepsilon_{1}-\frac{b_{1}\left(s+M_{3}\right)}{r}\right) T+\frac{b_{1}}{r} \ln \left(\frac{1}{1-\beta}\right) .
$$

For sufficiently small $\varepsilon_{1}>0$,

$$
\ln \gamma \approx\left(\hat{M}_{1}-\frac{b_{1}\left(s+M_{3}\right)}{r}\right) T+\frac{b_{1}}{r} \ln \left(\frac{1}{1-\beta}\right)-\ln \left(\frac{1}{1-\alpha}\right) .
$$

Since $\hat{M}_{1}<a_{1}-d_{1}$ and (9) hold, we can choose a small $n_{3}>0$ such that $\ln \gamma>0$ and, hence,

$$
\gamma \equiv(1-\alpha) \exp \left(\left(\hat{M}_{1}-b_{1} \varepsilon_{1}-\frac{b_{1}\left(s+M_{3}\right)}{r}\right) T+\frac{b_{1}}{r} \ln \left(\frac{1}{1-\beta}\right)\right)>1 .
$$

Then $B((k+i) T) \geq B(k T) \gamma^{i} \rightarrow \infty$ as $i \rightarrow \infty$. It is in contradiction to the boundedness of $B(t)$. Hence, there is $t_{1}>0$ such that $B\left(t_{1}\right) \geq n_{3}$.

Step 2. If $B(t) \geq n_{3}$ for all $t>t_{1}$, then the proof is complete. Otherwise, $B(t)<n_{3}$ for some $t>t_{1}$. Let $t^{*}=\inf _{t>t_{1}}\left\{B(t)<n_{3}\right\}$.

Case 1: $t^{*}=k_{1} T$ for some $k_{1} \in Z_{+}$. That is, for $t \in\left(t_{1}, t^{*}\right], B(t) \geq n_{3}$ and the continuity of $B(t)$ implies that $B\left(t^{*}\right)=n_{3}$.

Since there are $m>0$ and $n_{1}>0$ such that, for sufficiently large $t, B(t)<m$ and $n_{1}<C(t)<$ $m, m^{\prime}>0$ and $n_{1}^{\prime}>0$ are chosen so that

$$
B(t)<m^{\prime} \quad \text { and } \quad n_{1}^{\prime}<C(t)<m^{\prime}
$$

and

$$
\hat{M}_{1}<b_{1} m^{\prime}
$$

such that

$$
\left|\frac{1}{C\left(t^{*+}\right)}-\frac{\beta r}{\left(s+M_{3}\right)\left(1-\beta-e^{-\left(s+M_{3}\right) T}\right)}-\frac{r}{\left(s+M_{3}\right)}\right|-\beta<\frac{1}{n^{\prime}} .
$$

Then choose $k_{2}, k_{3} \in Z_{+}$such that

$$
k_{2} T>\frac{1}{\left(s+M_{3}\right)} \ln \left(\frac{\frac{1}{n^{\prime}}+\beta}{\varepsilon_{1}}\right)
$$


and

$$
(1-\alpha)^{k_{2}} \exp \left(\left(k_{2}+1\right) \gamma_{1} T\right) \gamma^{k_{3}}>1
$$

where

$$
\gamma_{1} \equiv \hat{M}_{1}-b_{1} m^{\prime}<0
$$

Let $T^{\prime}=k_{2} T+k_{3} T$. We claim that there is $t_{2} \in\left(t^{*}, t^{*}+T^{\prime}\right]$ such that $B\left(t_{2}\right)>n_{3}$. Otherwise, considering (17) with $\frac{1}{Z\left(t^{*+}\right)}=\frac{1}{C\left(t^{*+}\right)}$, we have

$$
\frac{1}{Z(t)}=\left(\frac{1}{Z\left(t^{*+}\right)}-\frac{\beta r}{\left(s+M_{3}\right)\left(1-\beta-e^{-\left(s+M_{3}\right) T}\right)}-\frac{r}{\left(s+M_{3}\right)}\right) e^{-\left(s+M_{3}\right)\left(t-t^{*}\right)}+\frac{1}{\tilde{Z}(t)}
$$

for $t \in(k T,(k+1) T]$ and $k_{1} \leq k \leq k_{1}+k_{2}+k_{3}$.

For $k_{2} T \leq t-t^{*} \leq T^{\prime}$, we have

$$
\begin{aligned}
\left|\frac{1}{Z(t)}-\frac{1}{\tilde{Z}(t)}\right| & =\left|\frac{1}{Z\left(t^{*+}\right)}-\frac{\beta r}{\left(s+M_{3}\right)\left(1-\beta-e^{-\left(s+M_{3}\right) T}\right)}-\frac{r}{\left(s+M_{3}\right)}\right| e^{-\left(s+M_{3}\right)\left(t-t^{*}\right)} \\
& =\left|\frac{1}{C\left(t^{*+}\right)}-\frac{\beta r}{\left(s+M_{3}\right)\left(1-\beta-e^{-\left(s+M_{3}\right) T}\right)}-\frac{r}{\left(s+M_{3}\right)}\right| e^{-\left(s+M_{3}\right)\left(t-t^{*}\right)} \\
& <\left(\frac{1}{n^{\prime}}+\beta\right) e^{-\left(s+M_{3}\right)\left(t-t^{*}\right)} \\
& <\left(\frac{1}{n^{\prime}}+\beta\right) e^{-\left(s+M_{3}\right) k_{2} T} \\
& <\varepsilon_{1} .
\end{aligned}
$$

Since (12), we have

$$
|Z(t)-\tilde{Z}(t)|<\frac{|Z(t)-\tilde{Z}(t)|}{|Z(t) \tilde{Z}(t)|}=\left|\frac{1}{Z(t)}-\frac{1}{\tilde{Z}(t)}\right|<\varepsilon_{1} .
$$

Then

$$
C(t) \leq Z(t)<\tilde{Z}(t)+\varepsilon_{1} .
$$

Similar to Step 1, we have

$$
\begin{aligned}
B\left(t^{*}+T^{\prime}\right) & =B\left(k_{1} T+k_{2} T+k_{3} T\right) \\
& \geq B\left(t^{*}+k_{2} T\right) \gamma^{k_{3}} .
\end{aligned}
$$

From (1a), we have

$$
\begin{aligned}
\frac{d B}{d t} & =a_{1} B\left(1-\frac{B}{h_{1}}\right)-\frac{b_{1} B C}{1+h_{2} B}-d_{1} B, \quad t \neq k T \\
& \geq\left(\hat{M}_{1}-b_{1} m^{\prime}\right) B=\gamma_{1} B
\end{aligned}
$$




$$
B\left(t^{+}\right)=(1-\alpha) B(t), \quad t=k T,
$$

and then integrating over $\left[t^{*}, t^{*}+k_{2} T\right]$, we obtain

$$
\begin{aligned}
B\left(t^{*}+k_{2} T\right) & \geq B\left(t^{*}\right)(1-\alpha)^{k_{2}} \exp \left(\int_{k_{1} T}^{k_{1} T+k_{2} T} \gamma_{1} d t\right) \\
& \geq n_{3}(1-\alpha)^{k_{2}} \exp \left(k_{2} \gamma_{1} T\right) \\
& \geq n_{3}(1-\alpha)^{k_{2}} \exp \left(\left(k_{2}+1\right) \gamma_{1} T\right)
\end{aligned}
$$

and hence

$$
\begin{aligned}
B\left(t^{*}+T^{\prime}\right) & \geq B\left(t^{*}+k_{2} T\right) \gamma^{k_{3}} \\
& \geq n_{3}(1-\alpha)^{k_{2}} \exp \left(\left(k_{2}+1\right) \gamma_{1} T\right) \gamma^{k_{3}} \\
& >n_{3} .
\end{aligned}
$$

Hence, the definition of $n_{3}$ is contradicted. Therefore, there exists $t_{2} \in\left(t^{*}, t^{*}+T^{\prime}\right]$ such that $B\left(t_{2}\right)>n_{3}$.

Now, let $\tilde{t}=\inf _{t>t^{*}}\left\{B(t)>n_{3}\right\}$. Then $B(t)<n_{3}$ for $t \in\left(t^{*}, \tilde{t}\right)$, and the continuity of $B(t)$ implies that $B(\tilde{t})=n_{3}$. Next, we choose $q \in Z_{+}$such that $q \leq k_{2}+k_{3}$ and $t^{*}+q T \geq \tilde{t}$, and suppose $t \in\left(t^{*}+(q-1) T, t^{*}+q T\right]$. From (25), we have

$$
\begin{aligned}
B(t) & \geq B\left(t^{*+}\right)(1-\alpha)^{q-1} \exp \left((q-1) \gamma_{1} T\right) \exp \left(\gamma_{1}\left(t-\left(t^{*}+(q-1) T\right)\right)\right) \\
& =B\left(t^{*}\right)(1-\alpha)^{q} \exp \left((q-1) \gamma_{1} T\right) \exp \left(\gamma_{1}\left(t-\left(t^{*}+(q-1) T\right)\right)\right) \\
& =n_{3}(1-\alpha)^{q} \exp \left(\gamma_{1}\left(t-t^{*}\right)\right) \\
& \geq n_{3}(1-\alpha)^{k_{2}+k_{3}} \exp \left(\gamma_{1} q T\right) \\
& \geq n_{3}(1-\alpha)^{k_{2}+k_{3}} \exp \left(\left(k_{2}+k_{3}\right) \gamma_{1} T\right) .
\end{aligned}
$$

We used $\gamma_{1}<0$ and $q \leq k_{2}+k_{3}$.

Letting

$$
\bar{n}_{2}=n_{3}(1-\alpha)^{k_{2}+k_{3}} \exp \left(\left(k_{2}+k_{3}\right) \gamma_{1} T\right)
$$

we can see that $B(t) \geq \bar{n}_{2}$ for $t \in\left(t^{*}, \tilde{t}\right)$. By using $\tilde{t}$ instead of $t^{*}$ and continuing in the same way, we then obtain $B(t) \geq \bar{n}_{2}$ for all $t$ large enough.

Case 2: $t^{*} \neq k T$ for all $k \in Z_{+}$. This implies that $B(t) \geq n_{3}$ for $t \in\left[t_{1}, t^{*}\right)$ and $B\left(t^{*}\right)=n_{3}$. For some $k_{1}^{\prime} \in Z_{+}$, suppose that $t^{*} \in\left(k_{1}^{\prime} T,\left(k_{1}^{\prime}+1\right) T\right)$.

Case 2.1: $B(t) \leq n_{3}$ for all $t \in\left(t^{*},\left(k_{1}^{\prime}+1\right) T\right]$. We claim that there is $t_{2}^{\prime} \in\left[\left(k_{1}^{\prime}+1\right) T,\left(k_{1}^{\prime}+\right.\right.$ 1) $\left.T+T^{\prime}\right]$ such that $x\left(t_{2}^{\prime}\right)>n_{3}$. Otherwise, considering (17) with $\frac{1}{Z\left(\left(k_{1}^{\prime}+1\right) T^{+}\right)}=\frac{1}{C\left(\left(k_{1}^{\prime}+1\right) T^{+}\right)}$. For $t \in(k T,(k+1) T], k_{1}^{\prime}+1 \leq k \leq k_{1}^{\prime}+1+k_{2}+k_{3}$, we obtain

$$
\begin{aligned}
\frac{1}{Z(t)}= & \left(\frac{1}{Z\left(\left(k_{1}^{\prime}+1\right) T^{+}\right)}-\frac{\beta r}{\left(s+M_{3}\right)\left(1-\beta-e^{-\left(s+M_{3}\right) T}\right)}-\frac{r}{\left(s+M_{3}\right)}\right) \\
& \times e^{-\left(s+M_{3}\right)\left(t-\left(k_{1}^{\prime}+1\right) T\right)}+\frac{1}{\tilde{Z}(t)} .
\end{aligned}
$$


For $k_{2} T \leq t-t^{*}$, as in Case 1 , we obtain

$$
|Z(t)-\tilde{Z}(t)|<\varepsilon_{1}
$$

Then

$$
C(t) \leq Z(t)<\tilde{Z}(t)+\varepsilon_{1} .
$$

Since $k_{2} T \leq\left(k_{1}^{\prime}+1+k_{2}\right) T-t^{*}$, we have

$$
\begin{aligned}
B\left(\left(k_{1}^{\prime}+1+k_{2}\right) T\right) & \geq B\left(t^{*}\right)(1-\alpha)^{k_{2}} \exp \left(\gamma_{1}\left(\left(k_{1}^{\prime}+1+k_{2}\right) T-t^{*}\right)\right) \\
& \geq n_{3}(1-\alpha)^{k_{2}} \exp \left(\gamma_{1}\left(\left(k_{1}^{\prime}+1+k_{2}\right) T-k_{1}^{\prime} T\right)\right) \\
& \geq n_{3}(1-\alpha)^{k_{2}} \exp \left(\left(k_{2}+1\right) \gamma_{1} T\right)
\end{aligned}
$$

Then

$$
\begin{aligned}
B\left(\left(k_{1}^{\prime}+1+k_{2}+k_{3}\right) T\right) & \geq B\left(\left(k_{1}^{\prime}+1+k_{2}\right) T\right) \gamma^{k_{3}} \\
& \geq n_{3}(1-\alpha)^{k_{2}} \exp \left(\left(k_{2}+1\right) \gamma_{1} T\right) \gamma^{k_{3}} \\
& >n_{3} .
\end{aligned}
$$

The definition of $n_{3}$ is contradicted and, hence, we can conclude that there is $t_{2}^{\prime} \in\left[\left(k_{1}^{\prime}+\right.\right.$ 1) $\left.T,\left(k_{1}^{\prime}+1\right) T+T^{\prime}\right]$ such that $B\left(t_{2}^{\prime}\right)>n_{3}$.

Now, let $\bar{t}=\inf _{t>t^{*}}\left\{B(t)>n_{3}\right\}$. Then $B(t) \leq n_{3}$ for $t \in\left[t^{*}, \bar{t}\right)$, and $B(\bar{t})=n_{3}$. We choose $q^{\prime} \in Z_{+}$such that $q^{\prime} \leq k_{2}+k_{3}+1$ and suppose $t \in\left(k_{1}^{\prime} T+\left(q^{\prime}-1\right) T, k_{1}^{\prime} T+q^{\prime} T\right]$. From (25), we have

$$
\begin{aligned}
B(t) & \geq B\left(\left(k_{1}^{\prime} T+\left(q^{\prime}-1\right) T\right)^{+}\right) \exp \left(\gamma_{1}\left(t-\left(k_{1}^{\prime} T+\left(q^{\prime}-1\right) T\right)\right)\right) \\
& =B\left(k_{1}^{\prime} T+\left(q^{\prime}-1\right) T\right)(1-\alpha) \exp \left(\gamma_{1}\left(t-\left(k_{1}^{\prime} T+\left(q^{\prime}-1\right) T\right)\right)\right) \\
& \geq B\left(t^{*}\right)(1-\alpha)^{q^{\prime}-1} \exp \left(\gamma_{1}\left(t-t^{*}\right)\right) \\
& \geq n_{3}(1-\alpha)^{q^{\prime}-1} \exp \left(\gamma_{1}\left(t-t^{*}\right)\right) .
\end{aligned}
$$

We used $\gamma_{1}<0$ and $t-t^{*} \leq q^{\prime} T$. Hence,

$$
B(t) \geq n_{3}(1-\alpha)^{k_{2}+k_{3}} \exp \left(\left(k_{2}+k_{3}+1\right) \gamma_{1} T\right) .
$$

Letting

$$
n_{2}=n_{3}(1-\alpha)^{k_{2}+k_{3}} \exp \left(\left(k_{2}+k_{3}+1\right) \gamma_{1} T\right)
$$

then, for $t \in\left(t^{*}, \bar{t}\right)$, we obtain $B(t) \geq n_{2}$. By using $\bar{t}$ instead of $t^{*}$ and continue with the same way, we shall obtain $B(t) \geq n_{2}$ for all $t$ large enough. 
Case 2.2: There is a $t^{\prime \prime} \in\left(t^{*},\left(k_{1}^{\prime}+1\right) T\right]$ such that $B\left(t^{\prime \prime}\right)>n_{3}$. Let $\underline{t}=\inf _{t>t^{*}}\left\{B(t)>n_{3}\right\}$. Hence, $B(t)<n_{3}$ for $t \in\left[t^{*}, \underline{t}\right)$, and $B(\underline{t})=n_{3}$. For $t \in\left[t^{*}, \underline{t}\right)$, (25) holds, we have

$$
\begin{aligned}
B(t) & \geq B\left(t^{*}\right) \exp \left(\int_{t^{*}}^{t} \gamma_{1} d t\right) \\
& =n_{3} \exp \left(\gamma_{1}\left(t-t^{*}\right)\right) \\
& \geq n_{3} \exp \left(\gamma_{1} T\right) \\
& >n_{2}
\end{aligned}
$$

since $t<k_{1}^{\prime} T+T<t^{*}+T$.

Since $B(\underline{t}) \geq n_{3}$, we can continue in the same way for $t>\underline{t}$. Since $n_{2}<\bar{n}_{2}<n_{3}$, we have $B(t) \geq n_{2}$ for $t \geq t_{1}$. The proof is complete.

\section{Existence of the positive periodic solution}

Let us investigate the possibility of positive periodic solution to the system (1a)-(1d) near $(0, \tilde{C}(t))$ by interchanging the state variables and consider the following system instead:

$$
\begin{aligned}
& \frac{d B}{d t}=a_{2} B\left(1-\frac{B}{h_{3}}\right)+\frac{r_{1} b_{1} B C}{1+h_{2} C}-d_{2} B, \quad t \neq k T, \\
& \frac{d C}{d t}=a_{1} C\left(1-\frac{C}{h_{1}}\right)-\frac{b_{1} B C}{1+h_{2} C}-d_{1} C, \quad t \neq k T,
\end{aligned}
$$

with

$$
\begin{array}{ll}
\Delta B(t)=-\beta B(t), & t=k T, \\
\Delta C(t)=-\alpha C(t), & t=k T .
\end{array}
$$

Let

$$
f_{1}(B, C)=a_{2} B\left(1-\frac{B}{h_{3}}\right)+\frac{r_{1} b_{1} B C}{1+h_{2} C}-d_{2} B, \quad f_{2}(B, C)=a_{1} C\left(1-\frac{C}{h_{1}}\right)-\frac{b_{1} B C}{1+h_{2} C}-d_{1} C .
$$

According to Lakmeche and Arini [13],

$$
\begin{aligned}
& \Theta_{1}(B, C)=(1-\beta) B, \quad \Theta_{2}(B, C)=(1-\alpha) C, \quad \varsigma(t)=(\tilde{C}(t), 0)^{T}, \\
& X_{0}=\left(\tilde{C}\left(\tau_{0}\right), 0\right)^{T}, \quad \tau_{0}=T_{1},
\end{aligned}
$$

and

$$
\begin{aligned}
& \frac{\partial \Phi_{1}\left(\tau_{0}, X_{0}\right)}{\partial \tau}=\frac{\partial \tilde{C}\left(\tau_{0}, X_{0}\right)}{\partial t}=\frac{\beta r \exp \left(-s \tau_{0}\right) \tilde{C}^{2}\left(\tau_{0}, X_{0}\right)}{1-\beta-\exp \left(-s \tau_{0}\right)}>0, \\
& \frac{\partial \Phi_{1}\left(\tau_{0}, X_{0}\right)}{\partial B}=\exp \left(\int_{0}^{\tau_{0}} \frac{\partial f_{1}(\zeta(u))}{\partial B} d u\right)>\frac{1}{1-\beta}>0 \\
& \frac{\partial \Phi_{1}\left(\tau_{0}, X_{0}\right)}{\partial C}
\end{aligned}
$$




$$
\begin{aligned}
& =\int_{0}^{\tau_{0}} \exp \left(\int_{v}^{\tau_{0}} \frac{\partial f_{1}(\varsigma(u))}{\partial B} d u\right) \frac{\partial f_{1}(\varsigma(v))}{\partial C} \exp \left(\int_{0}^{v} \frac{\partial f_{2}(\varsigma(u))}{\partial C} d u\right) d v \\
& =\int_{0}^{\tau_{0}} \exp \left(\int_{v}^{\tau_{0}}(s-2 r \tilde{C}(u)) d u\right)\left(r_{1} b_{1} \tilde{C}(v)\right) \\
& \times \exp \left(\int_{0}^{v}\left(a_{1}-d_{1}-b_{1} \tilde{C}(u)\right) d u\right) d v \\
& \frac{\partial \Phi_{2}\left(\tau_{0}, X_{0}\right)}{\partial C}=\exp \left(\int_{0}^{\tau_{0}} \frac{\partial f_{2}(\zeta(u))}{\partial C} d u\right)=\exp \left(\int_{0}^{\tau_{0}}\left(a_{1}-d_{1}-b_{1} \tilde{C}(u)\right) d u\right), \\
& \frac{\partial^{2} \Phi_{2}\left(\tau_{0}, X_{0}\right)}{\partial B \partial C} \\
& =\int_{0}^{\tau_{0}} \exp \left(\int_{v}^{\tau_{0}} \frac{\partial f_{2}(\varsigma(u))}{\partial C} d u\right) \frac{\partial^{2} f_{2}(\varsigma(v))}{\partial B \partial C} \exp \left(\int_{0}^{v} \frac{\partial f_{2}(\varsigma(u))}{\partial C} d u\right) d v \\
& =\frac{-b_{1} \tau_{0}}{1-\alpha}<0, \\
& \frac{\partial^{2} \Phi_{2}\left(\tau_{0}, X_{0}\right)}{\partial C^{2}} \\
& =\int_{0}^{\tau_{0}} \exp \left(\int_{v}^{\tau_{0}} \frac{\partial f_{2}(\varsigma(u))}{\partial C} d u\right) \frac{\partial^{2} f_{2}(\varsigma(v))}{\partial C^{2}} \exp \left(\int_{0}^{v} \frac{\partial f_{2}(\varsigma(u))}{\partial C} d u\right) d v \\
& +\int_{0}^{\tau_{0}}\left[\exp \left(\int_{v}^{\tau_{0}} \frac{\partial f_{2}(\varsigma(u))}{\partial C} d u\right) \frac{\partial^{2} f_{2}(\varsigma(v))}{\partial B \partial C}\right] \\
& \times\left[\int_{0}^{v} \exp \left(\int_{\theta}^{v} \frac{\partial f_{1}(\varsigma(u))}{\partial B} d u\right) \frac{\partial f_{1}(\varsigma(\theta))}{\partial C} \exp \left(\int_{0}^{\theta} \frac{\partial f_{2}(\varsigma(u))}{\partial C} d u\right) d \theta\right] d v \\
& =\int_{0}^{\tau_{0}}\left(-\frac{2 a_{1}}{h_{1}}+2 h_{1}\right) \exp \left(\int_{0}^{\tau_{0}}\left(a_{1}-d_{1}-b_{1} \tilde{C}(u)\right) d u\right) d v \\
& -b_{1} \int_{0}^{\tau_{0}}\left[\exp \left(\int_{v}^{\tau_{0}}\left(a_{1}-d_{1}-b_{1} \tilde{C}(u)\right) d u\right)\right] \\
& \times\left[\int_{0}^{v} \exp \left(\int_{\theta}^{v}(s-2 r \tilde{C}(u)) d u\right)\left(r_{1} b_{1} \tilde{C}(\theta)\right)\right. \\
& \left.\times \exp \left(\int_{0}^{\theta}\left(a_{1}-d_{1}-b_{1} \tilde{C}(u)\right) d u\right) d \theta\right] d v, \\
& \frac{\partial^{2} \Phi_{2}\left(\tau_{0}, X_{0}\right)}{\partial C \partial \tau} \\
& =\frac{\partial f_{2}\left(\varsigma\left(\tau_{0}\right)\right)}{\partial C} \exp \left(\int_{0}^{\tau_{0}} \frac{\partial f_{2}(\varsigma(u))}{\partial C} d u\right) \\
& =\left(a_{1}-d_{1}-b_{1} \tilde{C}\left(\tau_{0}\right)\right) \exp \left(\int_{0}^{\tau_{0}}\left(a_{1}-d_{1}-b_{1} \tilde{C}(u)\right) d u\right) \\
& =\frac{1}{1-\alpha}\left(a_{1}-d_{1}-\frac{b_{1} s\left(1-\beta-\exp \left(-s \tau_{0}\right)\right)}{\beta r \exp \left(-s \tau_{0}\right)+r\left(1-\beta-\exp \left(-s \tau_{0}\right)\right)}\right) \text {. }
\end{aligned}
$$

Now, we can compute

$$
d_{0}^{\prime}=1-\left(\frac{\partial \Theta_{2}}{\partial C} \frac{\partial \Phi_{2}}{\partial C}\right)_{\left(\tau_{0}, X_{0}\right)}=1-(1-\alpha) \exp \left(\int_{0}^{\tau_{0}}\left(a_{1}-d_{1}-b_{1} \tilde{C}(u)\right) d u\right)
$$


where $\tau_{0}$ is the root of $d_{0}^{\prime}=0$. Note that $d_{0}^{\prime}>0$ if $T<T_{1}$ and $d_{0}^{\prime}<0$ if $T>T_{1}$.

$$
a_{0}^{\prime}=1-\left(\frac{\partial \Theta_{1}}{\partial B} \frac{\partial \Phi_{1}}{\partial B}\right)_{\left(\tau_{0}, X_{0}\right)}=1-(1-\beta) \exp \left(\int_{0}^{\tau_{0}}(s-2 r \tilde{C}(u)) d u\right) .
$$

Note that $a_{0}^{\prime}>0$ if $T>T_{1}>T_{2}$,

$$
\begin{aligned}
b_{0}^{\prime}= & -\left(\frac{\partial \Theta_{1}}{\partial B} \frac{\partial \Phi_{1}}{\partial C}+\frac{\partial \Theta_{1}}{\partial C} \frac{\partial \Phi_{2}}{\partial C}\right)_{\left(\tau_{0}, X_{0}\right)} \\
= & -\frac{\partial \Theta_{1}}{\partial B} \frac{\partial \Phi_{1}\left(\tau_{0}, X_{0}\right)}{\partial C} \\
= & -(1-\beta) \int_{0}^{\tau_{0}} \exp \left(\int_{v}^{\tau_{0}}(s-2 r \tilde{C}(u)) d u\right)\left(r_{1} b_{1} \tilde{C}(v)\right) \\
& \times \exp \left(\int_{0}^{v}\left(a_{1}-d_{1}-b_{1} \tilde{C}(u)\right) d u\right) d v<0, \\
P^{*}= & -a_{1}+d_{1}+b_{1} s\left(\frac{1-\beta-\exp \left(-s \tau_{0}\right)}{\beta \exp \left(-s \tau_{0}\right)+\left(1-\beta-\exp \left(-s \tau_{0}\right)\right)}\right) \\
& \times\left(\frac{1}{r}+\frac{\tau_{0}(1-\beta) \beta s\left(1-\beta-\exp \left(-s \tau_{0}\right)\right)}{\left(1+\beta+\beta \exp \left(s \tau_{0}\right)\right)\left(\beta \exp \left(-s \tau_{0}\right)+1-\beta-\exp \left(-s \tau_{0}\right)\right)}\right), \\
Q^{*}= & 2(1-\alpha) \frac{b_{0}^{\prime}}{a_{0}^{\prime}} \frac{\partial^{2} \Phi_{2}}{\partial B \partial C}-(1-\alpha) \frac{\partial^{2} \Phi_{2}}{\partial C^{2}} .
\end{aligned}
$$

Note that $P^{*}<0$ and $Q^{*}>0$ if

$$
h_{1}^{2}<a_{1}
$$

and

$$
s^{2}+s-1<0
$$

Thus, $P^{*} Q^{*}<0$, and by Lakmeche and Arini [13], the following result is obtained.

Theorem 3 The system (25)-(28) has a positive periodic solution which is supercritical provided (2), (6), (9), (10), (12), (30), (31) hold, and $T>T_{1}>T_{2}$.

\section{Numerical simulations}

Figure 1 shows a simulation result of the system of Eqs. (1a)-(1d) with the parametric values $a_{1}=0.5, a_{2}=0.8, b_{1}=0.5, r_{1}=0.9, d_{1}=0.01, d_{2}=0.1, h_{1}=2, h_{2}=5, h_{3}=0.3, \alpha=$ $0.5, \beta=0.5, T=1, B(0)=5$, and $C(0)=10$ in which all the conditions in Theorem 1 are satisfied. The solution trajectory tends to a limit cycle as predicted in Theorem 1.

Figure 2 shows a simulation result of the system of equations (1a)-(1d) with the parametric values $a_{1}=0.5, a_{2}=0.7, b_{1}=0.5, r_{1}=0.9, d_{1}=0.01, d_{2}=0.1, h_{1}=3, h_{2}=5, h_{3}=0.2$, $\alpha=0.2, \beta=0.2, T=10, B(0)=5$, and $C(0)=5$ in which all the conditions in Theorem 2 are satisfied. The solution of the system shows permanence as predicted in Theorem 2 .

Figure 3 shows a simulation result of the system of equations (1a)-(1d) with the parametric values $a_{1}=0.5, a_{2}=0.4, b_{1}=0.5, r_{1}=0.9, d_{1}=0.01, d_{2}=0.2, h_{1}=0.3, h_{2}=2, h_{3}=0.5$, $\alpha=0.5, \beta=0.5, T=5, B(0)=5$, and $C(0)=5$ in which all the conditions in Theorem 3 are satisfied. The solution of the system is positive periodic as predicted in Theorem 3 . 


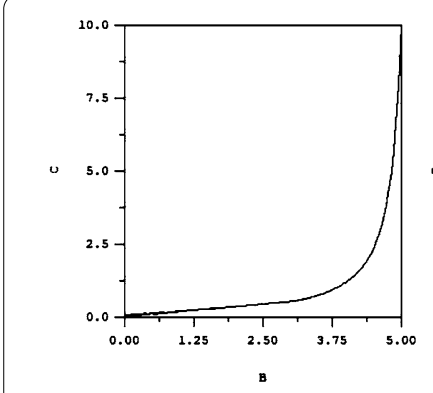

(a)

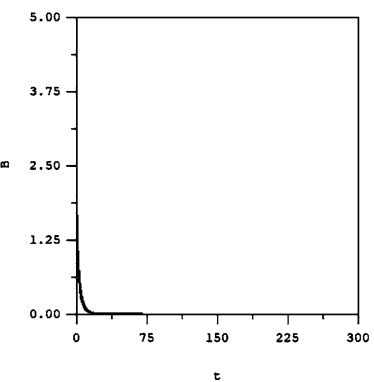

(b)

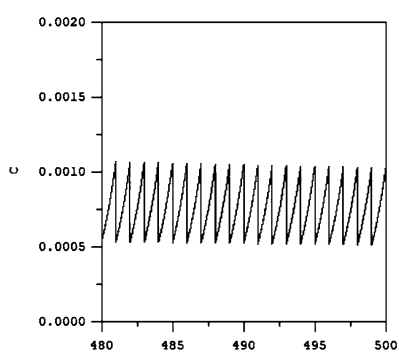

(c)

Figure 1 A computer simulation of Eqs. (1a)-(1d). The solution trajectory tends toward the oscillatory solution $(0, \tilde{C}(t))$ as time progresses. Here, $a_{1}=0.5, a_{2}=0.8, b_{1}=0.5, r_{1}=0.9$,

$d_{1}=0.01, d_{2}=0.1, h_{1}=2, h_{2}=5, h_{3}=0.3, \alpha=0.5, \beta=0.5, T=1, B(0)=5$, and $C(0)=10$. Here, all conditions in Theorem 1 are satisfied. (a) The solution trajectory projected on the $(B, C)$-plane. (b) The time series of the population density of brown planthoppers (B) tending to a vanishing level. (c) The time series of the population density of Cyrtorhinus lividipennis (C) exhibiting positive oscillation

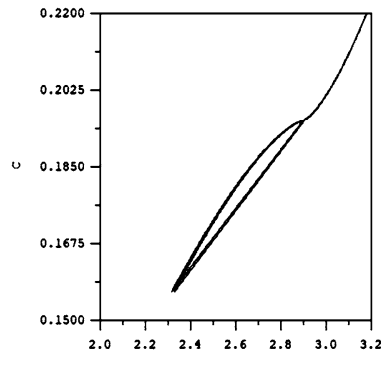

(a)

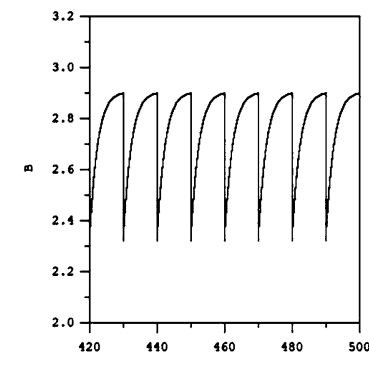

(b)

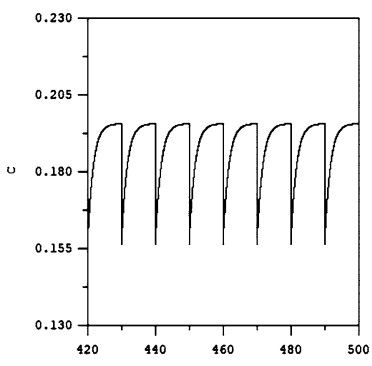

(c)

Figure 2 A computer simulation of Eqs. (1a)-(1d). The solution of the system is permanent. Here, $a_{1}=0.5, a_{2}=0.7, b_{1}=0.5, r_{1}=0.9, d_{1}=0.01, d_{2}=0.1, h_{1}=3, h_{2}=5, h_{3}=0.2, \alpha=0.2, \beta=0.2, T=10, B(0)=5$, and $C(0)=5$. Here, all conditions in Theorem 2 are satisfied. (a) The solution trajectory projected on the $(B, C)$-plane. (b) The bounded time series of the population density of brown planthoppers (B). (c) The bounded time series of the population density of Cyrtorhinus lividipennis (C)

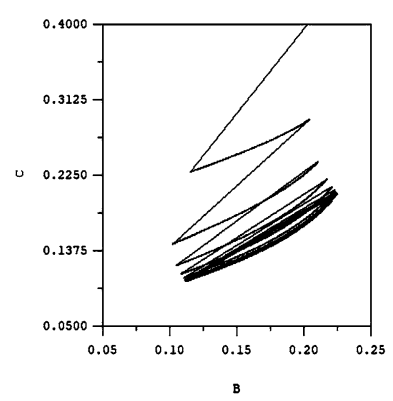

(a)

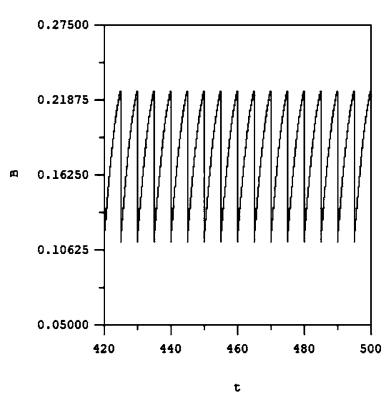

(b)

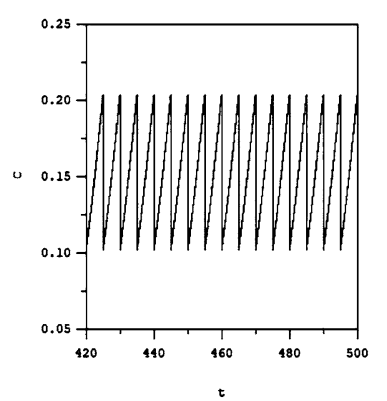

(c)

Figure 3 A computer simulation of Eqs. (1a)-(1d). The solution of the system is positive periodic. Here, $a_{1}=0.5, a_{2}=0.4, b_{1}=0.5, r_{1}=0.9, d_{1}=0.01, d_{2}=0.2, h_{1}=0.3, h_{2}=2, h_{3}=0.5, \alpha=0.5, \beta=0.5, T=5, B(0)=5$, and $C(0)=5$. Here, all conditions in Theorem 3 are satisfied. (a) The solution trajectory projected on $(B, C)$-plane. (b) The time series of the population density of brown planthoppers (B) and (c) The time series of population density of Cyrtorhinus lividipennis (C) 


\section{Conclusion}

We investigate the dynamics behaviors of the populations of BPH and Cyrtorhinus lividipennis when insecticide is utilized to control the population of $\mathrm{BPH}$ in the paddy field through an impulsive mathematical model. Once insecticide is applied, both BPH and Cyrtorhinus lividipennis populations decrease rapidly. The appropriate duration $T$ between two consecutive applications of insecticide might lead to effective control (the population of BPH reaches the vanishing level or maintains a level lower than the desired level) of BPH while Cyrtorhinus lividipennis still survive in the paddy field.

\section{Funding}

We acknowledge the support by the Centre of Excellence in Mathematics, the Commission on Higher Education, Thailand.

\section{Competing interests}

The authors declare that they have no competing interests.

\section{Authors' contributions}

All authors contributed equally to this work. All authors read and approved the final manuscript.

\section{Author details}

1 Department of Mathematics, Mahasarakham University, Mahasarakham, Thailand. ${ }^{2}$ Center of Excellence in Mathematics, Commission on Higher Education, Bangkok, Thailand. ${ }^{3}$ Department of Mathematics, Faculty of Science, Mahidol University, Bangkok, Thailand.

\section{Publisher's Note}

Springer Nature remains neutral with regard to jurisdictional claims in published maps and institutional affiliations.

Received: 31 January 2019 Accepted: 3 May 2019 Published online: 14 May 2019

\section{References}

1. Stout, M.J.: Chapter I: Host-plant resistance in pest management. In: Abrol, D.P. (ed.) Integrated Pest Management Elsevier, Amsterdam (2014)

2. Escalada, M.M., Luecha, M., Heong, K.L.: Chapter 10-Social impacts of planthopper outbreaks: case study from Thailand. In: Heong, K.L., Cheng, J.A., Escalada, M.M. (eds.) Rice Planthoppers: Ecology, Management, Socio Economics and Policy, pp. 209-226. Zhejiang University Press, Hangzhou (2015). https://doi.org/10.1007/978-94-017-9535-7

3. Huang, J., Qiao, F., Zhang, L., Rozelle, S.: Farm Pesticide, Rice Production and Human Health, Centre for Chinese Agricultural Policy. Chinese Academy of Sciences, Beijing (2000)

4. Rola, A.C., Pingali, P.L.: Pesticides, Rice Productivity, and Farmers' Health: An Economic Assessment. International Rice Research Institute and World Resource Institute, Washington (1993)

5. Zhang, X., Liao, X., Mao, K., Zhang, K., Wan, H., Li, J.: Insecticide resistance monitoring and correlation analysis of insecticides in field populations of the brown planthopper Nilaparvara lugens (Stål) in China 2012-2014. Pestic. Biochem. Physiol. 132, 13-20 (2016)

6. Ramli, N.H., Yusup, S., Kueh, B.W.B., Kamarulzaman, P.S.D., Osman, N., Rahim, M.A., Aziz, R., Mokhtar, S., Ahmad, A.B.: Effectiveness of biopesticides against brown planthopppers (Nilaparvata lugens) in paddy cultivation. Sustainable Chemistry and Pharmacy 8, 16-20 (2018)

7. Katti, G., Pasalu, I.C., Padmakumari, A.P., Padmavathi, C., Jhansilakshmi, V., Krishnaiah, N.V., Bentur, J.S., Prasad, J.S., Rao, Y.K.: Biological control of insect pests of rice. Technical Bulletin, Directorate of Rice Research, Rajendranagar, Hyderabad, AP, India 22, 22 (2007)

8. Sigsgaard, L.: Early season natural control of the brown planthopper Nilaparvata lugens: the contribution and interaction of two spider species and a predatory bug. Bull. Entomol. Res. 97, 533-544 (2007)

9. Preetha, G., Stanley, J., Suresh, S., Samiyappan, R.: Risk assessment of insecticides used in rice on miridbug, Cyrtorhinus lividipennis Reuter, the important predator of brown planthopper, Nilaparvata lugens (Stal.). Chemosphere 80, 498-503 (2010)

10. Lu, Z., Yu, X., Hu, C.: Predatory behavior of mirid bug, Cyrtorhinus lividipennis, on rice plants with different nitrogen regimes. International Rice Research Notes. 29, 38-40 (2004)

11. Liu, B., Zhi, Y., Chen, L.: The dynamics of a predator-prey model with Ivlev's functional response concerning integrated pest management. Acta Math. Appl. Sin. 20(1), 133-146 (2004)

12. Lakshmikantham, V., Bainov, D.D., Simeonov, P.S.: Theory of Impulsive Differential Equations. World Scientific, Singapore (1989)

13. Lakmeche, A., Arini, O.: Bifurcation of non trivial periodic solutions of impulsive differential equations arising chemotherapeutic treatment. Dyn. Contin. Discrete Impuls. Syst., Ser. A Math. Anal. 7, 265-287 (2000) 